\title{
TROCLEOPLASTIA POR RESSECÇÃO ASSOCIADA À TRANSPOSIÇÃO DA TUBEROSIDADE TIBIAL E SOBREPOSIC̄ÃO DA FÁSCIA LATA PARA O TRATAMENTO DA LUXAÇÃO PATELAR EM CÃES
}

\author{
Jose Ademar Villanova Junior ${ }^{1}$, Vinícius Ferreira Caron ${ }^{1}$ \\ ${ }^{1}$ Pontifícia Universidade Católica do Paraná, Campus São José dos Pinhais/PR - jose.villanova@pucpr.br
}

\begin{abstract}
RESUMO: Neste estudo retrospectivo é relatada a experiência com a técnica cirúrgica composta de trocleoplastia por ressecção associada à transposição da tuberosidade tibial e sobreposição da fáscia lata no tratamento da luxação patelar em cães. Vinte e sete cães adultos, de ambos os sexos, participaram deste estudo. Os animais apresentavam diferentes graus de luxação patelar, após a intervenção cirúrgica foram acompanhados por período que variou de três meses a dois anos. Apenas um teve recorrência da luxação. $O$ retorno à função do membro afetado variou de uma semana a quatro meses de período pós-operatório. A reconstituição cirúrgica foi de fácil execução e os resultados obtidos neste estudo retrospectivo permitem concluir que a tática cirúrgica empregada é apropriada para luxações patelares, tanto mediais quanto laterais.
\end{abstract}

Palavras-chave: cães; cirurgia ortopédica veterinária; deslocamento patelar; eixo fêmuro-tíbiopatelar; reconstituição cirúrgica

\section{TROCHLEOPLASTY FOR RESECTION ASSOCIATED WITH THE TRANSPOSITION OF THE TIBIAL TUBEROSITY AND OVERLAPPING OF THE FASCIA LATA FOR TREATMENT OF THE PATELLAR LUXATION IN DOGS}

ABSTRACT: In this retrospective study we reported the experience with the surgical technique consisted of resection by trochleoplasty associated with transposition of tibial tuberosity and overlaps the fascia lata in the treatment of patellar luxation in dogs. Twenty-seven adult dogs of both sexes participated in this study, they had different degrees of patellar dislocation after surgery were followed up for period ranging from three months to two years, of which only one had recurrence of dislocation, the return the function of the affected limb ranged from one week to four months postoperatively. The surgical repair was easy and the results of this retrospective study showed that the surgical procedure employed is appropriate for patellar dislocations, as both medial side.

Key words: dogs; patellar dislocation; shaft femur-tibial-patellar;surgical reconstruction; veterinary orthopedic surgery 


\section{INTRODUÇÃO}

Denominada como osso sesamóide, a patela está situada distalmente ao tendão do músculo quadríceps femoral e inserida a tuberosidade tibial através do ligamento patelar. Participa da articulação fêmuro-tíbio-patelar e internamente articula-se com a tróclea femoral, caracterizando assim a luxação patelar como uma afecção poliostóstica (Vasseur, 2007). Em conjunto com o músculo quadríceps femoral, a patela é primordial ao movimento extensor do joelho. $O$ perfeito alinhamento entre 0 quadríceps femoral, a patela, a tróclea femoral, o ligamento patelar e a tuberosidade tibial é o que proporciona o movimento congruente. Em cães normais, o ângulo de projeção frontal, traçado do trocânter até a diáfise femorais é de $135^{\circ}$ a $145^{\circ}$ (Olsmtead, 1995). Alterações nas estruturas anatômicas decorrentes da luxação medial da patela, como a coxa vara, diminuem o ângulo da diáfise femoral em relação à fossa trocantérica (Padilha Filho et al., 2005; Souza et al., 2009). Na luxação patelar lateral, ocorre a coxa valga e aumento do ângulo diafisário femoral (Hayes et al., 1994, Denny e Butterworth, 2006).

Segundo Schultz (2008), existe numerosas técnicas cirúrgicas que visam restringir a patela ao sulco troclear. A transposição da tuberosidade tibial, liberação de restrição medial, reforço de restrição lateral, aprofundamento do sulco troclear, osteotomia femoral e osteotomia tibial têm sido reinvindicados para correção de luxação patelar. Em geral, é necessária combinação de técnicas. As técnicas utilizadas dependem da gravidade da luxação, presença de deformidades esqueléticas e preferência do cirurgião.

A trocleoplastia é técnica que aprofunda a tróclea rasa, ausente ou convexa. A transposição da tuberosidade tibial objetiva recolocar esta estrutura mais cranialmente no membro, isto ajudará na estabilidade patelar. A sobreposição da fáscia lata, promoverá redução da lassidão articular (Brinker et al., 1997).

De acordo com Denny e Butterworth (2006) o prognóstico depende da graduação da lesão, da técnica cirúrgica utilizada e dos cuidados no período pósoperatório, como a restrição de espaço e administração correta de medicação.

O objetivo deste estudo retrospectivo foi relatar a eficácia da técnica cirúrgica composta de trocleoplastia por recessão associada à transposição da tuberosidade tibial e sobreposição da fáscia lata no tratamento da luxação patelar em cães.

\section{MATERIAL E MÉTODOS}

Foram submetidos à técnica cirúrgica composta de trocleoplastia por recessão associada à transposição da tuberosidade tibial e sobreposição da fáscia lata 27 cães portadores de luxação patelar, destes 20 (74\%) possuíam raça; $21(77,8 \%)$ pesavam até 10kg; 21 $(77,8 \%)$ tinham luxação medial; oito (29,6\%) apresentavam luxação patelar grau II, $13(48,1 \%)$ grau III e seis $(22,2 \%)$ eram grau IV.

Constatado o deslocamento patelar e definido o grau da afecção (graus II, III ou IV), os animais foram radiografados para detecção de doença articular degenerativa e análise da posição da tuberosidade tibial. Para aqueles animais que apresentavam claudicação frequente foi indicada a intervenção cirúrgica.

O procedimento cirúrgico iniciou-se com a incisão cutânea realizada em dupla curva tendo início no quarto distal do fêmur, desenvolvendo-se sobre a crista troclear lateral, paralelamente à patela. Ao nível da metade do ligamento patelar, a incisão mudou de direção, para cruzar o ligamento num ângulo de $45^{\circ}$, e terminou 
distalmente a superfície medial da tuberosidade tibial. $\mathrm{O}$ tecido celular subcutâneo foi seccionado da mesma maneira, divulsionado e as abas cutâneas afastadas.

$\mathrm{Na}$ sequência foi efetuada incisão através da fáscia da articulação fêmurotíbio-patelar, propositalmente foi deixada bastante fáscia na borda da patela, para receber suturas, quando a articulação for ocluída. A seguir foi promovida incisão parapatelar, através da cápsula articular. Com a articulação estendida, a patela e a musculatura adjacente foram medialmente ou lateralmente luxadas, mantendo o sentido da luxação presente. O afastamento da cápsula articular expôs integralmente o interior da articulação.

$$
\text { Acessada a articulação, }
$$

primeiramente foi realizada a técnica da ressecção da cunha troclear para 0 restabelecimento da estabilidade patelofemoral, nesta técnica incisões foram efetuadas com a ajuda de bisturi com lâmina n.15, tendo início nas cristas trocleares, após as incisões a cunha cartilaginosa foi temporariamente retirada, o osso subjacente foi removido, mediante o uso de limas circulares, a cunha foi então reposicionada na reentrância, permitindo o assentamento mais profundo da patela no interior da tróclea, a pressão patelar manteve a cunha cartilaginosa na tróclea femoral. Em seguida foi realizada a técnica de transposição da tuberosidade tibial, nesta técnica foi elevado o músculo tibial cranial em direção ao platô tibial, e com o auxílio de uma pequena serra, a tuberosidade tibial foi osteotomizada e transposta centralmente, de modo que a patela e o músculo quadríceps foram realinhados com o fêmur e a tíbia. A região que recebeu a tuberosidade sofreu remoção do osso cortical e exposição do osso esponjoso, a tuberosidade foi fixada com um parafuso ortopédico 316L (Figura 1).

Por último foi realizada imbricação fascial, evitando acúmulo de material excedente. Esta técnica consiste na feitura de sutura em dois planos, sendo o primeiro aplicado para tração fáscial, aproximando os músculos de suas aponeuroses, em seguida uma sobresutura completa a imbricação, ambas feitas com Poliglactina 910, n. 3-0 a 4-0, com padrão de sutura de Lembert interrompido (Figura 2). O tecido celular subcutâneo foi suturado com sutura de cushing, usando Poliglactina 910, n. 2-0 a 3-0, e a pele com fio mononáilon 3-0 em pontos isolados simples.

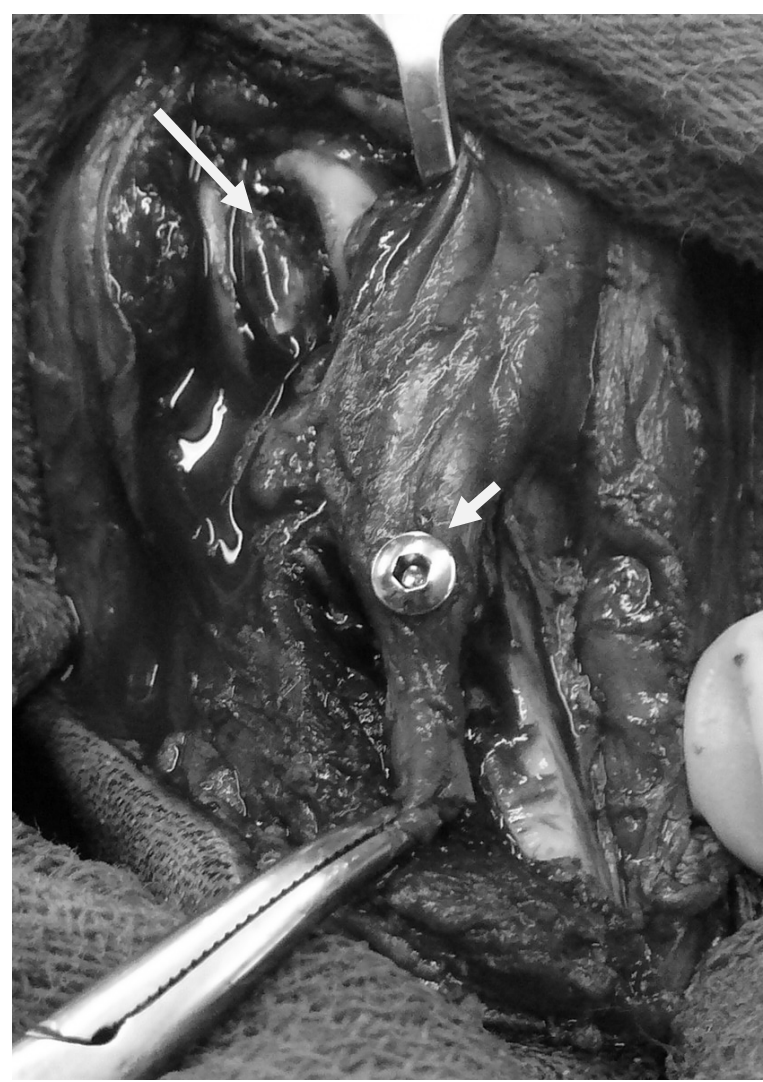

Figura 1 - Joelho canino após trocleoplastia por recessão e reposicionamento da cunha cartilaginosa (seta maior) e transposição da tuberosidade tibial fixada com parafuso ortopédico 316L (seta menor).

No período pós-operatório foi imposta moderação de atividades físicas durante seis semanas. Foi feito o uso de ataduras objetivando dar efeito compressivo e limitação da flexão do joelho. Como medicações pósoperatórias foram administrados meloxicam $(0,1 \mathrm{mg} / \mathrm{kg}$ a cada 24 horas) durante três a cinco dias e enrofloxacina 
Tabela 1 - Características da deambulação, em graus correspondentes, para avaliação clínica pré e pós-operatória de cães submetidos à trocleoplastia por ressecção associada à transposição da tuberosidade tibial e sobreposição da fáscia lata.

\begin{tabular}{|c|c|}
\hline GRAU & CARACTERISTICAS \\
\hline I & Uso e apoio do membro \\
\hline II & $\begin{array}{l}\text { Apresenta uso e apoio infreqüentes do membro durante estação e ao caminhar. } \\
\text { Sustentação do peso na extremidade afetada, elevando-a ao correr. }\end{array}$ \\
\hline III & $\begin{array}{l}\text { Apresenta uso claudicante do membro em estação e ao caminhar. Sustenta } \\
\text { parcialmente o peso, elevando o membro ao correr. }\end{array}$ \\
\hline IV - A & $\begin{array}{l}\text { Caminha sem claudicar e posiciona-se normalmente em estação. Claudica ao correr } \\
\text { sem elevar o membro. }\end{array}$ \\
\hline $\begin{array}{l}\text { IV }-\mathrm{B} \\
\mathrm{V}\end{array}$ & $\begin{array}{l}\text { Claudica ao caminhar e ao correr, porém, não eleva o membro. } \\
\text { Apresenta uso funcional do membro }\end{array}$ \\
\hline
\end{tabular}

( $5 \mathrm{mg} / \mathrm{kg}$ a cada 12 horas) durante sete a dez dias.

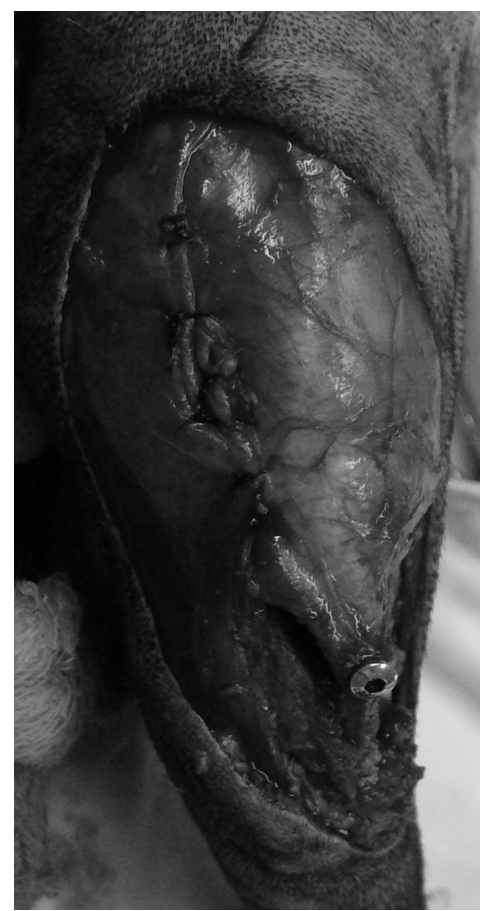

Figura 2 - Joelho canino após transposição da tuberosidade tibial e embricamento da cápsula articular. Observar realinhamento do eixo fêmuro-tíbio-patelar.

A avaliação clínica da recuperação funcional do membro teve por base os graus de recuperação apresentados na Tabela 1, modificada de Tudury e Raiser (1985). O acompanhamento pósoperatório foi realizado com sete, 15, 45 e 120 dias. Ao término de cada período pós-operatório, os animais eram avaliados quanto a qualidade da deambulação e se havia ou não recorrência da luxação patelar.

\section{RESULTADOS E DISCUSSÃO}

Embora a luxação patelar seja reconhecida como uma doença de desenvolvimento, não há consenso sobre sua patogênese. Sabe-se que é o resultado de anormalidades anatômicas envolvendo o eixo fêmuro-tíbio-patelar (Harasen, 2006a). Visando restabelecer o movimento congruente e retificar as irregularidades ósseas, as técnicas de artroplastia aplicáveis na estabilização de luxações patelares podem ser divididas em duas classes: reconstrução do tecido mole e reconstrução óssea. Julgamento considerável e experiência são necessários para decidir o melhor procedimento ou combinação de procedimentos para um caso particular (Brinker et al., 1997; Harasen, 2006b).

Um princípio cardeal é que a deformidade esquelética, tal como o desvio da tuberosidade tibial e sulco troclear raso, deve ser corrigida por técnicas de reconstrução óssea. A tentativa de superar tal má formação esquelética por reconstrução de tecido mole somente, é a maior causa de insucesso. Procedimentos no tecido mole, somente, devem ser limitados a casos de grau I. A falha na transposição da tuberosidade tibial é talvez a maior causa de insucesso (Brinker et al., 1997).

A técnica cirúrgica praticada neste estudo abordou tecidos duros (trocleo- 
Tabela 2 - Avaliação dos graus de deambulação nos períodos correspondentes ao pré-operatório e aos 7, 15, 45 e 120 dias de pós-operatório de cães submetidos à trocleoplastia por ressecção associada à transposição da tuberosidade tibial e sobreposição da fáscia lata.

\begin{tabular}{|c|c|c|c|c|c|}
\hline \multirow[t]{2}{*}{$\begin{array}{l}\text { Número } \\
\text { do cão }\end{array}$} & \multirow{2}{*}{$\begin{array}{c}\text { Graus das } \\
\text { características de } \\
\text { deambulação -pré- } \\
\text { operatório }\end{array}$} & \multicolumn{4}{|c|}{$\begin{array}{c}\text { Graus das características de deambulação relacionados aos } \\
\text { dias de avaliação pós-operatória }\end{array}$} \\
\hline & & 7 & 15 & 45 & 120 \\
\hline 1 & III & & & V & \\
\hline 2 & II & & V & & \\
\hline 3 & II & & & IV-A & \\
\hline 4 & III & & & V & \\
\hline 5 & III & & IV-A & & \\
\hline 6 & II & & & V & \\
\hline 7 & III & & V & & \\
\hline 8 & III & & & & V \\
\hline 9 & II & & & V & \\
\hline 10 & II & & IV-A & & \\
\hline 11 & III & III & III & III & III \\
\hline 12 & III & & & IV-A & \\
\hline 13 & III & & & V & \\
\hline 14 & III & IV-A & & & \\
\hline 15 & III & & IV-A & & \\
\hline 16 & III & & IV-B & & \\
\hline 17 & II & V & & & \\
\hline 18 & II & & IV-B & & \\
\hline 19 & III & & $\mathrm{V}$ & & \\
\hline 20 & III & & V & & \\
\hline 21 & II & & & IV-A & \\
\hline 22 & III & & & & V \\
\hline 23 & III & & & & V \\
\hline 24 & II & & & V & \\
\hline 25 & III & & IV-B & & \\
\hline 26 & III & & & & V \\
\hline 27 & III & & V & & \\
\hline
\end{tabular}

Modificada de Tudury e Raiser (1985).

plastia por ressecção e transposição da tuberosidade tibial) e tecido mole (embricamento da cápsula articular), sendo de fácil execução e pouco onerosa.

A Tabela 2 mostra a melhora progressiva da deambulação e que apenas em um $(3,7 \%)$ dos 27 animais estudados houve recorrência, este era portador de luxação patelar grau IV, tal resultado corrobora com Popak Maria et al. (2001), que cita o prognóstico favorável para graus II e III e desfavorável para grau IV.

Dos 27 animais estudados, 19 $(70,4 \%)$ deles possuíam grau III de deambulação pré-operatória, o restante $(29,6 \%)$ era grau II; passados sete dias do ato operatório, dois $(7,4 \%)$ animais mostraram melhora, pois passaram a ser graus IV-A e V; com 15 dias de pósoperatório, outros $11(40,7 \%)$ mostraram graus de melhora; aos 45 dias de pósoperatório $22(81,5 \%)$ dos animais operados apresentaram boa deambulação e aos 120 dias de observação pós-operatória, 26 (96,3\%) passaram a ter graus mínimos a moderados de claudicação, sendo sete $(25,9 \%)$ graus IV-A (caminha sem claudicar e posiciona-se normalmente em estação), três (11,1\%) grau IV-B (claudica ao caminhar e ao correr, porém, não eleva o membro) e 16 $(59,3 \%)$ animais graus $\mathrm{V}$ (apresenta uso funcional do membro). 


\section{CONCLUSÃO}

Mediante os resultados obtidos nesse estudo, é possível concluir que a técnica cirúrgica composta de trocleoplastia por recessão, transposição da tuberosidade tibial e sobreposição da fáscia lata é pouco onerosa, pois necessita após osteotomia tibial de apenas um implante para restabelecimento do eixo fêmurotíbio-patelar; também é tecnicamente fácil, apesar de envolver abordagem em tecidos duros e tecido mole, e na maioria das situações, permite recuperar a funcionalidade da articulação do joelho.

\section{REFERÊNCIAS}

BRINKER, W.O; PIERMATTEI, D.L.; FLO, G.L. Handbook of Small Animal Orthopedics and Fracture Repair, 3. ed., Pennsylvania: Saunders, p.516-552, 1997.

DENNY, H.R.; BUTTERWORTH, S.J. Cirurgia Ortopédica em Cães e Gatos. 4.ed. São Paulo: Editora Roca, p. 401-406, 2006.

HARASEN, G. Patellar luxation: Pathogenesis and surgical correction. The Canadian Veterinary Journal, v.47 n.10, p.1037-1039, 2006a.

HARASEN, G. Patellar luxation. The Canadian Veterinary Journal, v.47 n.8, p.817-818, 2006b.
HAYES, A.G.; BOUDRIEAU, R.J.;

HUNGERFORD, L.L. Frequency and distribution of medial and lateral patellar luxation in dogs: 124 cases (1982-1992). Journal of the American Veterinary Medical Association, v.205, n.5, p.716-720, 1994.

OLMSTEAD, M.L. Small Animal Orthopedics. Missouri: Mosby, p.395-404, 1995.

PADILHA FILHO, J.G.; DÓREA NETO, F.A.; SANCHES, R.C. et al. Treatment of the patellar luxation in toy poodles. Ciência Rural, v.35, n.4, p.843-847, 2005.

POPAK MARIA, P.; PADILHA FILHO, J.G.;

ALMEIDA, T.L. Luxação medial de patela em cães - revisão. Clínica Veterinária, n.34, p.25-32, 2001.

SCHULTZ, K. Afecções Articulares. In: FOSSUM, T.W. Cirurgia de Pequenos Animais. 3.ed. São Paulo: Elsevier, 2008. p.1289-1299.

SOUZA, M.M.D.; RAHAL, S.C.; OTONI, C.C. et al. Luxação de patela em cães: estudo retrospectivo. Arquivos Brasileiros de Medicina Veterinária e Zootecnia, v.61, n.2, p.523-526, 2009.

TUDURY, E.A.; RAISER, A.G. Redução de fraturas distais do femur de cães, empregando dois pinos de Steinmann em substituição aos de Rush. Revista do Centro de Ciências Rurais, v.15, n.2, p.141-155, 1985. VASSEUR, P.B. Articulação do Joelho. In: SLATTER, D. Manual de Cirurgia de Pequenos Animais. 3.ed. São Paulo: Editora Manole, 2007. p.2122-2126. 